\title{
Evolution of Plant Growth and Defense in a Continental Introduction
}

\author{
Anurag A. Agrawal, ${ }^{1,2, \star}$ Amy P. Hastings, ${ }^{1}$ Gideon S. Bradburd, ${ }^{3}$ Ellen C. Woods, ${ }^{1, \dagger}$ Tobias Züst, ${ }^{1}$ \\ Jeffrey A. Harvey, ${ }^{4}$ and Tibor Bukovinszky ${ }^{5}$
}

1. Department of Ecology and Evolutionary Biology, Cornell University, Ithaca, New York 14853; 2. Department of Entomology, Cornell University, Ithaca, New York 14853; 3. Center for Population Biology, Department of Evolution and Ecology, University of California, Davis, California 95616; 4. Department of Terrestrial Ecology, Netherlands Institute of Ecology, 6708 PB Wageningen, The Netherlands; 5. Resource Ecology Group, Wageningen University, Droevendaalsesteeg 3, 6708 PB Wageningen, The Netherlands

Submitted October 31, 2014; Accepted March 4, 2015; Electronically published May 4, 2015

Online enhancements: appendixes. Dryad data: http://dx.doi.org/10.5061/dryad.vr176.

\begin{abstract}
AвsтRACT: Substantial research has addressed adaptation of nonnative biota to novel environments, yet surprisingly little work has integrated population genetic structure and the mechanisms underlying phenotypic differentiation in ecologically important traits. We report on studies of the common milkweed Asclepias syriaca, which was introduced from North America to Europe over the past 400 years and which lacks most of its specialized herbivores in the introduced range. Using 10 populations from each continent grown in a common environment, we identified several growth and defense traits that have diverged, despite low neutral genetic differentiation between continents. We next developed a Bayesian modeling approach to account for relationships between molecular and phenotypic differences, confirming that continental trait differentiation was greater than expected from neutral genetic differentiation. We found evidence that growth-related traits adaptively diverged within and between continents. Inducible defenses triggered by monarch butterfly herbivory were substantially reduced in European populations, and this reduction in inducibility was concordant with altered phytohormonal dynamics, reduced plant growth, and a trade-off with constitutive investment. Freedom from the community of native and specialized herbivores may have favored constitutive over induced defense. Our replicated analysis of plant growth and defense, including phenotypically plastic traits, suggests adaptive evolution following a continental introduction.
\end{abstract}

Keywords: Cardenolides, common milkweed Asclepias syriaca, ecological genetics, monarch butterfly Danaus plexippus, latex, plantinsect interactions.

\footnotetext{
* Corresponding author; e-mail: aa337@cornell.edu.

${ }^{\dagger}$ Present address: Department of Ecology and Evolutionary Biology, University of Connecticut, Storrs, Connecticut 06269.

Am. Nat. 2015. Vol. 186, pp. E1-E15. (c) 2015 by The University of Chicago. 0003-0147/2015/18601-55860\$15.00. All rights reserved. DOI: $10.1086 / 681622$
}

\section{Introduction}

As Charles Elton observed more than 50 years ago, transcontinental species introductions offer remarkable natural experiments to test hypotheses about the ecology and evolution of organismal traits (Sax et al. 2005). Several hypotheses have been put forward to explain why some plants exhibit dramatically altered growth and defense traits following introduction to new continents. First, plants may adaptively evolve in response to the novel biotic environment, specifically, to the lack of coevolved enemies or competitors (Blossey and Nötzold 1995; Sakai et al. 2001; Callaway et al. 2004; Orians and Ward 2010). Second, they may rapidly adapt to abiotic conditions (Maron et al. 2004; Colautti et al. 2009); for example, when species are transported across continents, populations in the introduced range often evolve adaptive latitudinal clines in their phenotype, mirroring the clines of their native range (Colautti et al. 2009). Finally, founder effects may reduce genetic diversity, exacerbate genetic drift, or constrain adaptation in introduced populations (Barrett and Husband 1990; Bossdorf et al. 2005; Kolbe et al. 2012). Of course, these effects are not mutually exclusive, and thus unraveling the drivers of continental differentiation requires an integrative approach.

Common milkweed (Asclepias syriaca L.) is an excellent model to study the evolutionary dynamics of continental introduction. In the native range, $A$. syriaca shows adaptive phenotypic clines along its broad latitudinal distribution in eastern North America (Woods et al. 2012). The species was repeatedly introduced to Europe over the past several centuries (as early as $1630 \mathrm{CE}$ ) both as an ornamental plant and for several practical uses (Gaertner 1979). In fact, Carl Linnaeus kept the species epithet "syriaca" because, at the time of his work, the plant was already com- 
mon in central Europe and was sometimes mistaken to be the same species as a milkweed native to Syria (Gaertner 1979). Asclepias syriaca has two well-characterized antiherbivore defenses (latex and cardenolides), both of which are inducible following herbivore attack and neither of which has any role in the primary metabolism of the plant (Agrawal and Konno 2009; Agrawal et al. 2012). Because A. syriaca is attacked by a range of predominantly specialist herbivores (including larvae of the monarch butterfly; Agrawal 2005), most of which occur only in North America, we hypothesized that enemy escape in Europe has resulted in the evolution of reduced levels of defense.

In this study, we first characterized the phenotypic traits of 10 North American and 10 European A. syriaca populations (table 1; fig. 1) in a controlled environment. Specifically, we tested whether plant growth-related traits (seed mass, root and shoot biomass accumulation), foliar traits (specific leaf area, water content, carbon : nitrogen ratio, trichrome density), and constitutive or induced defense traits (latex production and cardenolide concentration with or without previous damage by monarch larvae) have differentiated between North American and European populations. In order to test whether phenotypic differentiation was adaptive (greater than that predicted by genetic drift), we studied the genetic structure of populations on both continents through analysis of polymorphic microsatellite DNA markers.

This study makes several advances building on past work integrating population genetic structure with phenotypic evolution in continental introductions. First, we implemented a Bayesian statistical method that estimates phenotypic differentiation between the continents after accounting for the genetic covariance structure at neutral loci among populations. We compared these results with a more traditional test (the ratio of $Q$ to $F$ statistics; Whitlock 2008), which has a different set of assumptions (see "Methods"). Second, we examined the consistency of climatic clines in driving phenotypes on both continents, taking a first step toward partitioning the role of abiotic versus biotic drivers of evolutionary change across continents. Finally, in addition to traditional plant growth and defensive traits, we tested for differentiation in the inducibility of defense, a set of traits that cannot be evaluated when plants are grown under a single (undamaged) environmental condition (Cipollini et al. 2005; Eigenbrode et al. 2008; Carrillo et al. 2012; Wang et al. 2013). Given that our analyses revealed continental differentiation in induced defense, we conducted a set of ecophysiological experiments to evaluate three hypothesized but non-mutually exclusive mechanisms that could explain such differential inducibility:

Table 1: Population information for the 10 introduced European populations and 10 native North American populations of common milkweed Asclepias syriaca

\begin{tabular}{lclllrlrrr}
\hline Population & MAT & MAP & Latitude & Longitude & $N$ & $H_{\mathrm{O}}$ & $H_{\mathrm{E}}$ & $N_{\mathrm{A}}$ & $F_{\mathrm{IS}}$ \\
\hline Bologna, Italy & 11.535 & 65.08 & 44.49651 & 11.34338 & 7 & .182 & .201 & 1.455 \\
Érd, Hungary & 10.11 & 43.75 & 47.37696 & 18.92052 & 11 & .488 & .458 & 2.909 & -.083 \\
Kisszékely, Hungary & 10.2 & 46.295 & 46.67889 & 18.53943 & 8 & .489 & .458 & 2.818 & -.101 \\
Malacky R, Slovakia & 9.255 & 48.83 & 48.40278 & 17.01889 & 8 & .489 & .349 & 2 & -.449 \\
Malacky G, Slovakia & 9.255 & 48.83 & 48.39639 & 17.01556 & 8 & .5 & .445 & 2.182 & -.176 \\
Neszür, Hungary & 9.66 & 44.835 & 47.46674 & 19.85006 & 8 & .386 & .386 & 3 & -.023 \\
Stretavka, Slovakia & 6.93 & 47.42 & 48.62528 & 22.00083 & 10 & .536 & .399 & 2.455 & -.369 \\
Stroglach, Austria & 6.99 & 79 & 46.66 & 14.47 & 8 & .136 & .156 & 1.364 \\
Troyes, France & 10.62 & 57.67 & 48.29781 & 4.086914 & 5 & .2 & .232 & 1.636 \\
Vojany, Slovakia & 8.8725 & 57.9725 & 48.55194 & 21.98389 & 8 & .42 & .398 & 2.545 & -.097 \\
Anderson, IN & 10.31 & 80.83 & 40.1 & -85.6833 & 8 & .477 & .461 & 2.909 & -.080 \\
Chapel Hill, NC & 14.445 & 94.54 & 35.96667 & -79.1 & 5 & .636 & .537 & 2.818 & -.263 \\
East Lansing, MI & 8.32 & 64.67 & 42.7348 & -84.4808 & 8 & .466 & .515 & 3.000 & .056 \\
Fulks Run, VA & 11.56 & 79.5 & 38.66667 & -78.9 & 8 & .523 & .446 & 3.000 & -.218 \\
Hanover, NH & 7.5 & 73.17 & 43.7 & -72.2833 & 8 & .489 & .470 & 3.000 & -.062 \\
Ithaca, NY & 7.715 & 74.165 & 42.43333 & -76.5 & 9 & .444 & .425 & 3.091 & -.053 \\
Philipsburg, PA & 9.345 & 82.875 & 40.91667 & -78.05 & 8 & .489 & .489 & 3.273 & -.039 \\
Phoenixville, PA & 10.675 & 96.04 & 40.1 & -75.4667 & 8 & .489 & .473 & 3.273 & -.068 \\
Sylvania, OH & 9.27 & 70.33 & 41.71667 & -83.7 & 9 & .556 & .434 & 2.545 & -.312 \\
Urbana, IL & 10.76 & 81.58 & 40.11667 & -88.2 & 9 & .475 & .481 & 3.636 & -.009 \\
\hline
\end{tabular}

Note: MAT, mean annual temperature $\left({ }^{\circ} \mathrm{C}\right)$; MAP, mean annual precipitation $(\mathrm{cm}) ; N$, number of individuals sampled for genetic analysis; $H_{\mathrm{O}}$, mean observed heterozygosity; $H_{\mathrm{E}}$, mean expected heterozygosity; $N_{\mathrm{A}}$, mean number of alleles; $F_{\mathrm{IS}}$, inbreeding coefficient. 

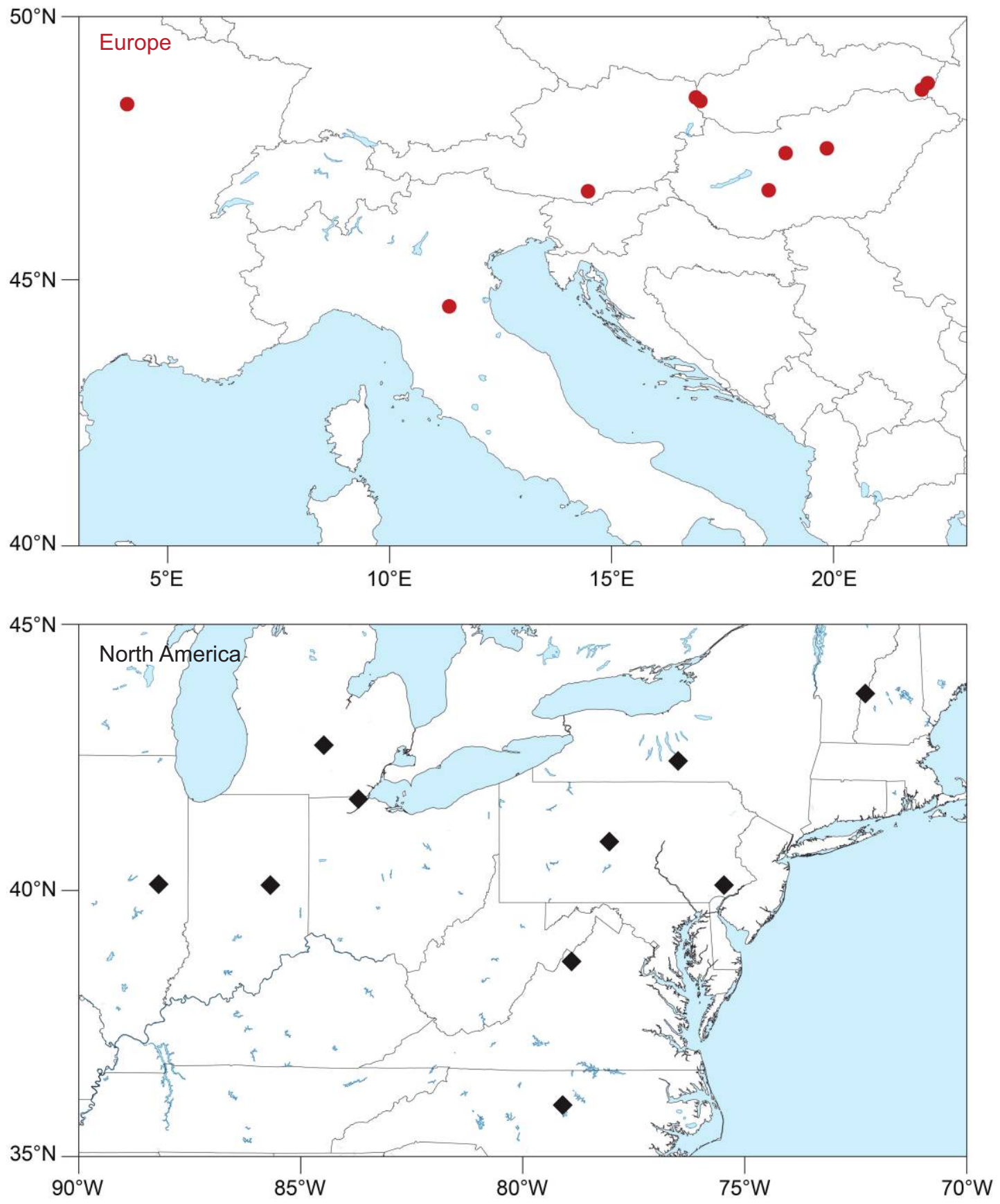

Figure 1: Locations of Asclepias syriaca seed collections in North America and Europe.

changes in phytohormonal induction, plant growth rate, and trade-offs between constitutive and induced responses. Overall, this work represents an integrated approachspanning ecophysiology, species interactions, and population genetics - to understand evolution following a continental introduction.

\section{Methods}

\section{Population Collections}

To compare genetically based traits across continents while controlling for variation caused by latitudinal clines, we (and colleagues; see "Acknowledgments") collected seeds 
from 20 natural populations, 10 from each continent. Most populations occurred in old fields or along roadsides and were sampled along eastern North America and central Europe, spanning several degrees of latitude on each continent (fig. 1; table 1). We collected seeds from three to five maternal plants separated by at least a few meters in each population.

Populations from the two continents differed in mean latitude (on average, European populations are from latitudes $7^{\circ}$ farther north; $\left.F_{1,18}=82.64, P<.001\right)$. We collected four environmental measures on the 20 populations from the National Oceanic and Atmospheric Administration (http://www.noaa.gov) and the Intergovernmental Panel on Climate Change (http://www.ipcc-data.org), following methods outlined by Montague et al. (2008) and using climate stations in closest proximity to the collection sites. All stations conformed to World Meteorological Organization standards. Mean annual temperature (MAT), number of days during which the maximum temperature is $\leq 0^{\circ} \mathrm{C}$, and growing degree units (an estimate of growing season developed for agricultural crops) were all strongly correlated with each other (and with latitude; Woods et al. 2012), but none were correlated with site precipitation. Thus, we selected two independent predictors, MAT and mean annual precipitation (MAP; see also Woods et al. 2012). We controlled for MAT in our sampling scheme such that it did not differ between the groups of populations across the two continents $\left(F_{1,18}=0.650, P=.431\right)$; mean precipitation, however, was $29 \%$ lower among the European compared with North American populations $\left(F_{1,18}=11.84, P=.003\right)$. Nonetheless, site-specific precipitation was not a significant predictor in any of the initial analyses and was hence dropped from the final models.

\section{Assessment of Plant Traits}

We assessed seed mass by individually weighing 10 randomly selected seeds collected from each of the 10 populations on each of the two continents. We grew two cohorts of plants in a walk-in growth chamber $(350 \mu \mathrm{E}$ of light; $14 \mathrm{~L}: 10 \mathrm{D}$ cycle at $27^{\circ}$ and $25^{\circ} \mathrm{C}$, respectively; $40 \%-$ $50 \%$ humidity). In the first cohort (2010), a total of 268 plants from the 20 populations were grown, with 12-16 plants per population. For each population, we mixed seeds from at least three maternal collections. Seeds were treated with $5 \%$ bleach, scarified, stratified at $4^{\circ} \mathrm{C}$ for 7 days, germinated at $28^{\circ} \mathrm{C}$, and grown in potting soil $(10-\mathrm{cm}$-diameter pots). One week after planting, plants were given a single application of dilute fertilizer $(\mathrm{N}: \mathrm{P}: \mathrm{K}$ ratio $21: 5: 20$, 150 ppm $\mathrm{N}[\mu \mathrm{g} / \mathrm{g}])$.

Because all plants were started from seed collected from wild populations, we cannot exclude maternal effects from contributing to the phenotypes measured in the growth chambers. However, given the wide geographical range of populations, within-continent variation in most climatic variables was on average equal to or higher than betweencontinent variation; thus, such effects are likely to have imposed minimal biases on the observed continental differentiation. Indeed, factors other than temperature, growing season length, and precipitation would have to consistently differ across the continents for maternal effects to have produced the observed phenotypic differentiation between continents. One measure of maternal allocation - seed masswas measured on individual seeds from all populations (see "Results"). Although MAT of collection sites affected seed mass, there were no continental differences in this trait. In addition, our past studies on defensive traits of common milkweed have shown that family mean traits measured on young plants (as in this experiment) hold true into the second year of growth (Agrawal 2005; Woods et al. 2012), suggesting that short-lived maternal effects do not substantially affect genotypic differences between plants.

After 25 days of growth, half of the plants from each population were randomly assigned to be exposed to feeding by a freshly hatched monarch caterpillar from a laboratory colony. Caterpillars were removed and weighed after 4 days, which is sufficient time to induce plant defenses of milkweed (A. A. Agrawal, unpublished data). After an additional day, using standardized methods detailed elsewhere (Agrawal 2005), we measured specific leaf area (SLA; area/ dry mass), water content, trichrome density, foliar carbon: nitrogen ratio $(\mathrm{C}: \mathrm{N})$, and latex exudation. For latex, we cut the tip $(2-3 \mathrm{~mm})$ off of the youngest fully expanded leaf and collected the exuded latex on a preweighed $1-\mathrm{cm}$ disc of filter paper. After absorbing all the latex, we placed the disc into a preweighed microcentrifuge tube. These tubes were stored in a freezer briefly until they could be reweighed to estimate the amount of latex collected. On the opposite leaf from which latex was collected, a hole punch (6-mm diameter) was taken from the tip of the leaf and weighed fresh; this same leaf disc was used for trichome counts under a dissecting microscope and was then dried at $40^{\circ} \mathrm{C}$ for assessment of dry mass. Both leaves were then collected for assessment of $\mathrm{C}, \mathrm{N}$, and cardenolides; leaves were frozen and then oven dried at $40^{\circ} \mathrm{C}$. Plant growth was assessed as total above- and belowground biomass at the end of the experiment. Roots were carefully washed free of soil, and all plant parts were dried at $40^{\circ} \mathrm{C}$ and weighed. In addition, we counted the number of stem buds produced on roots (an estimate of clonal reproductive potential) and calculated the root: shoot ratio for all plants.

Following methods of Bingham and Agrawal (2010), foliar cardenolide concentrations were assessed by highperformance liquid chromatography in a subset of seven to eight replicates from each population (total $n=156$ ) of the first cohort. In the second cohort (2012), 187 plants 
were grown as above from the same original seed stock and were again treated with caterpillar damage or left as controls. After the damaging caterpillars were removed, these plants were split into two groups, one for a subsequent monarch caterpillar bioassay to assess induced resistance (113 plants, with two to three plants per treatment and population; measured as the mass gain of the second set of caterpillars after 3 days of feeding) and the other for an assay of phytohormones (64 plants representing a subset of nine North American and seven European populations as a result of inadvertent plant losses during the experiment). For the hormone analysis, we employed an established liquid chromatography-mass spectrometry procedure (Thaler et al. 2010). Further detailed methods for cardenolides and hormones are provided in appendix A (apps. A and B available online). Population means for all phenotypic data are available in the Dryad Digital Repository: http://dx.doi.org /10.5061/dryad.vr176 (Agrawal et al. 2015b).

\section{Approach to Analyses of Phenotypes}

We conducted most initial analyses in two stages. First, we conducted nested ANOVAs with population (random effect) nested within continent and continent (fixed effect); where appropriate, the herbivore induction treatment and continent $x$ induction interactions were also included in the model. Analyses were conducted using restricted maximum likelihood in JMP. In a follow-up analysis for each trait, we used the population mean phenotype for the analysis, with continent as a fixed effect and MAT of the collection sites as a covariate. In all analyses, the statistical tests for continental differentiation had $n=20$. To address trade-offs between constitutive and induced responses to herbivory, we used a bias-corrected Monte Carlo procedure that accounts for sampling variation, measurement error, and induced susceptibility (Morris et al. 2006), followed by Carrillo et al.'s (2012) extension of this approach to address differences in trade-offs between the continents. To address type I error in analysis of multiple plant traits, we employed the binomial expansion test (Zar 1996).

\section{DNA Extraction, PCR, Genotyping}

Two sets of leaf tissue were collected for genetic analysis; the first consisted of four plants from each of the 20 populations in the first cohort of plants (see above), and the second consisted of one to seven plants per population in the second cohort. Both cohorts were from the same set of field-collected seeds. The total data set consisted of 161 individuals, with five to 11 plant samples per population. For both collections, a single piece of leaf tissue $\left(\approx 1 \mathrm{~cm}^{2}\right)$ was sampled from each plant and immediately frozen at $-80^{\circ} \mathrm{C}$. The tissue was then freeze dried and ground into powder using a Retsch Mixer Mill, and DNA was extracted using a standard cetyltrimethylammonium bromide (CTAB)-chloroform protocol. Briefly, genomic DNA was obtained by incubating samples at $60^{\circ} \mathrm{C}$ in CTAB buffer containing betamercaptoethanol for $20 \mathrm{~min}$ and then extracting with chloroform. DNA was precipitated from the aqueous phase with isopropanol, washed with ethanol, dried, and resuspended in Tris-EDTA buffer. Each sample was genotyped for 11 published microsatellite markers in two multiplex polymerase chain reaction (PCR) processes using the Type-It Microsatellite PCR Kit (Qiagen, Valencia, CA): AsyrB102, B5, C102, C109, C124, C4, A106, B121, and C103 from O'Quinn and Fishbein (2009); and AS94, ASF9 from Kabat et al. (2010). PCR reactions contained $1 \mu \mathrm{L}$ of diluted genomic DNA and were set up according to the kit protocol (with Q-solution) but scaled down to a $10 \mu \mathrm{L}$ total volume. PCR followed a touchdown protocol of $95^{\circ} \mathrm{C}$ for $5 \mathrm{~min}$, followed by 28 cycles of $95^{\circ} \mathrm{C}$ for $30 \mathrm{~s}, 60^{\circ} \mathrm{C}$ for $90 \mathrm{~s}$, and $72^{\circ} \mathrm{C}$ for $30 \mathrm{~s}$ (the annealing temperature decreased by $1^{\circ} \mathrm{C}$ each cycle for the first seven cycles), with a final extension at $60^{\circ} \mathrm{C}$ for $30 \mathrm{~min}$. Products were diluted 1:3 and analyzed on a 3730xl DNA Analyzer (Applied Biosystems, Life Technologies, Grand Island, NY) at the Cornell University Life Sciences Core Laboratories Center. Allele sizes were determined using Genemapper (ver. 3.5; Applied Biosystems), with all calls checked by eye.

\section{Population Genetic Structure}

We performed exact tests of Hardy-Weinberg equilibrium (locus-by-locus option; 100,000 dememorizations; 1,000,000 steps per Markov chain) and pairwise linkage disequilibrium among loci $(10,000$ permutations, two initial conditions for EM algorithm) on our microsatellite data set in Arlequin (ver. 3.5.1.2; Excoffier and Lischer 2010). After applying a sequential Bonferroni correction for multiple comparisons, no loci were found to deviate significantly from Hardy-Weinberg equilibrium, and a single linkage disequilibrium test was found to be significant between one pair of loci in a single population (AsyrB5 and AsyrC124, Cierna Voda, Slovakia; $P=.0002$ ). Standard population genetics statistics (observed and expected heterozygosity, allelic richness and inbreeding coefficients) were estimated using Microsatellite Analyzer version 4.05 (Dieringer and Schlotterer 2003).

We used Arlequin to calculate pairwise $F_{\mathrm{ST}}$ values (Slatkin's distance, $F_{\mathrm{ST}}$-like, 10,000 permutations) for the 10 populations on each continent and to implement an AMOVA, using number of different alleles as a distance measure $\left(F_{\mathrm{ST}^{-}}\right.$ like, 16,000 permutations) on the entire data set (all 20 populations). This analysis allowed us to partition genetic variance into within-population, among-population, and between-continent components and to calculate fixation in- 
dices for each component. In addition, we ran an AMOVA on each continent separately in order to calculate global $F_{\mathrm{ST}}$ values for comparison with $Q$ statistics (see below). In order to assess neutrality among microsatellites, an outlier test (Grubb's test) was performed on average $F_{\mathrm{ST}}$ values for each locus within each continent. No outliers were identified in either analysis.

We used Structure 2.3.2 (Pritchard et al. 2000) to identify clusters with distinct allele frequencies and assign individuals to these clusters without a priori knowledge of source populations. We ran the admixture model with correlated allele frequencies, with 500,000 Markov chain Monte Carlo generations after a burn-in period of 30,000 generations (all parameters had stabilized). In order to identify the appropriate number of clusters for our data set, we performed independent runs at $k$ from 1 to 20, with 10 runs at each value of $k$, and then examined the rate of change in the log probability using the approach of Evanno et al. (2005). We averaged the cluster assignment coefficient matrices for the 10 runs at $k=3$ using CLUMPP 1.1.2 (full search algorithm; Jakobsson and Rosenberg 2007) and used Distruct 1.1 (Rosenberg 2004) to visualize overall population structure.

\section{Tests of Adaptation}

To test for a signal of adaptation in our phenotypic data while accounting for potential nonindependence due to different amounts of shared evolutionary history between the different populations, we used two approaches. Our first approach involved a statistical model adapted from Grafen (1989) and similar to that of Ovaskainen et al. (2011). Here we modeled the data as random multivariate normally distributed variables, with means estimated separately for each continent, an estimated contribution to the mean phenotype from MAT, and a covariance structure proportional to the population allelic covariance matrix. Specifically, we used the model

$$
\begin{aligned}
D \sim M V N(\text { mean } & =\beta_{\mathrm{E}} \times \xi+\beta_{\mathrm{NA}} \times \eta+\beta_{\text {Enviro }} \times \varepsilon, \\
\text { covariance } & =\alpha \times \Omega+\sigma \times I),
\end{aligned}
$$

where $D$ is the vector of phenotypic measurements across all $k$ populations; $\xi$ is a Boolean vector of length $k$ that indexes the continent of origin of each population ( 1 if the population is from Europe, 0 if it is not); $\eta$ is a similar vector of length $k$ but with a 1 indexing populations from North America and a 0 for populations from Europe; $\beta_{\mathrm{E}}$ and $\beta_{\mathrm{NA}}$ are the phenotypic means estimated for European and North American populations, respectively; $\varepsilon$ is a vector of length $k$ of a normalized climatic or environmental variable measured in each population; $\beta_{\text {Enviro }}$ is the parameter that governs the contribution of that variable to the phenotypic mean; $\Omega$ is the $k \times k$ population allelic covariance matrix; $\alpha$ is the scalar coefficient of that genetic covariance matrix; $\sigma$ is a scalar giving the population variance; and $I$ is the $k \times k$ identity matrix. We note that this modeling scheme, in which Europe and North America are assigned separate means, is equivalent to modeling a single mean for both continents, with a second parameter modeling the departure in a single continent from that mean. A cell in the population allelic covariance matrix, $\Omega$, was derived by first calculating the per-locus allele sharing for a pair of individuals (one from each population) averaged across all loci and then averaging that measurement across all pairs of individuals from the two populations. This procedure was repeated for all population pairs to populate the full $\Omega$ matrix. This matrix is calculated from finite data and, as such, may be a noisy estimate of the true population covariance. The elements $D, \xi, \eta, \varepsilon, \Omega$, and $I$ are observed; the elements $\beta_{\mathrm{E}}, \beta_{\mathrm{NA}}, \beta_{\mathrm{Enviro}}, \alpha$, and $\sigma$ are estimated as part of the model.

We took a Bayesian approach to inference on this problem and specified a prior on each parameter estimated as part of the model. We modeled $\beta_{\mathrm{E}}, \beta_{\mathrm{NA}}$, and $\beta_{\text {Enviro }}$ as uniform, with a minimum at -100 and a maximum at 100 $(\sim U(-100,100))$, and $\alpha$ and $\sigma$ as exponentials with mean $1(\sim \exp (\lambda=1))$. The expression for the posterior probability of the parameters of the model is therefore given by

$$
\begin{gathered}
P\left(\beta_{\mathrm{E}}, \beta_{\mathrm{NA}}, \beta_{\text {Enviro }}, \alpha, \sigma \mid D\right) \propto \\
P\left(D \mid \beta_{\mathrm{E}}, \beta_{\mathrm{NA}}, \beta_{\text {Enviro }}, \alpha, \sigma\right) \times P\left(\beta_{\mathrm{E}}\right) \times P\left(\beta_{\mathrm{NA}}\right) \\
\times P\left(\beta_{\text {Enviro }}\right) \times P(\alpha) \times P(\sigma),
\end{gathered}
$$

where the proportionality is up to the normalization constant given by the right-hand side of the equation (the likelihood multiplied by the priors) integrated over all parameters.

To estimate the posterior distribution of our parameters, we implemented a custom Markov chain Monte Carlo sampler and executed it in the statistical platform R (R Development Core Team 2007). In all analyses, both the phenotypic and MAT data were mean centered and normalized by their standard deviation. All analyses were run for 10 million generations, with multiple, independent runs initiated from random locations in parameter space to ensure convergence on the stationary distribution.

Conceptually, this model assumes that neutral genetic divergence, as summarized by $\Omega$, determines expectations of the correlation structure of neutral phenotypic divergence between populations. Our approach is a variant on the classic animal model of quantitative genetics (for a review, see Wilson et al. 2010) and is similar to that of Viss- 
cher et al. (2006) or Lachmuth et al. (2011) in that it uses genetic relationships rather than pedigree information to predict phenotypic covariance between sampled populations. By explicitly modeling this genetic structure, we are ensuring that any signal of differentiation between $\beta_{\mathrm{E}}$ and $\beta_{\mathrm{NA}}$ is consistent with a hypothesis of continental adaptation rather than simply the result of different amounts of shared evolutionary history. We assessed statistical significance using $95 \%$ credible intervals of the joint marginal difference between $\beta_{\mathrm{E}}$ and $\beta_{\mathrm{NA}}$. We interpret an overlap with 0 as an inability to reject the null hypothesis that the means for European and North American populations are not significantly different, while a $95 \%$ credible interval that does not contain 0 is strong evidence that the phenotypic means for the two continents are significantly different. Inference scripts used in analyses are available on GitHub at https:// github.com/gbradburd/pheno_cov.

In the second, more traditional approach, we examined the ratio of differentiation of plant phenotypic traits $\left(Q_{\mathrm{ST}}\right)$ to neutral molecular differentiation $\left(F_{\mathrm{ST}}\right)$ between populations. Such an approach has been used to show adaptive differentiation in some but not other plant introductions (Chun et al. 2009, 2011). Although the $Q / F$ approach is similar to the Bayesian multivariate modeling approach described above - both seek to identify phenotypic differentiation above and beyond that predicted by neutral genetic differentiation - they are different enough to merit inclusion of both analyses. Specifically, the calculation of $F_{\mathrm{CT}}$ and $Q_{\mathrm{CT}}$ is naive to the genetic relationships between sampled populations and therefore treats populations as independent when, in fact, their differing amounts of shared evolutionary history mean that they probably are not. This heterogeneity in shared genetic drift may bias $Q / F$ results. In contrast, our multivariate approach explicitly accounts for the amount of shared genetic drift between populations within and between continents in the correlation structure of the phenotypic data and is thus robust to differing patterns of population structure. In addition, the multivariate approach, like all Bayesian approaches, offers a simple and intuitive way to accommodate uncertainty in parameter estimates. Other limitations of the $Q / F$ approach have been outlined by Whitlock (2008). We therefore include both tests here but present the details of the $Q / F$ analyses in appendix B.

\section{Results}

ANOVA on growth and defense traits of plants from the 20 populations grown in a common environment revealed that 10 of the 15 traits significantly diverged between continents (table A1; tables A1, A2 available online). This level of continental differentiation prompted our analysis of population genetic structure and subsequent tests of adaptation.

\section{Neutral Genetic Differentiation}

Analysis of population differentiation within continents revealed stronger structure in Europe (mean pairwise $F_{\mathrm{ST}}=$ 0.309 ) versus North America (mean $F_{\mathrm{ST}}=0.103$; see percent of variation among populations in table 2). Consistent with this finding, European samples were more minimally admixed with respect to three identified genetic clusters (fig. A1; figs. A1-A3 available online).

European populations of Asclepias syriaca were characterized by reduced within-population heterozygosity $\left(H_{\text {obs: }}\right.$ : $24 \%$ reduction, $F_{1,18}=5.69, P=.028 ; H_{\text {exp }}: 26 \%$ reduction, $F_{1,18}=11.42, P=.003$ ) and allelic richness (mean num-

Table 2: AMOVA for the full data set as well as each continent separately

\begin{tabular}{|c|c|c|c|c|}
\hline Source of variation & df & SS & Variance components & Percentage of variation \\
\hline \multicolumn{5}{|l|}{ Both continents: } \\
\hline Among continents & 1 & 20.023 & .04951 & $1.70^{*}$ \\
\hline Among populations within continents & 18 & 211.837 & .59121 & $20.25^{* * *}$ \\
\hline Within populations & 302 & 688.152 & 2.27865 & $78.05^{* * *}$ \\
\hline Total & 321 & 920.012 & 2.91937 & \\
\hline \multicolumn{5}{|l|}{ North America: } \\
\hline Among populations & 9 & 65.797 & .29616 & $10.29^{* * *}$ \\
\hline Within populations & 150 & 387.315 & 2.58210 & $89.71^{\mathrm{a}}$ \\
\hline Total & 159 & 453.113 & 2.87826 & \\
\hline \multicolumn{5}{|l|}{ Europe: } \\
\hline Among populations & 9 & 146.040 & .88290 & $30.85^{* * *}$ \\
\hline Within populations & 152 & 300.837 & 1.97919 & $69.15^{\mathrm{a}}$ \\
\hline Total & 161 & 446.877 & 2.86209 & \\
\hline
\end{tabular}

\footnotetext{
a Indicates no significance test because all remaining variation is included in this term.

${ }^{*} P<.05$.

${ }^{* * *} P<.0001$.
} 
ber of alleles per locus: $27 \%$ reduction, $F_{1,18}=14.73, P=$ .001) compared with native North American populations. Nonetheless, inbreeding coefficients $\left(F_{\text {IS }}\right)$ did not differ $\left(F_{1,18}=0.007, P=.937\right)$ and were slightly negative for both continents, consistent with the largely self-incompatible nature of this species (for population means, see table 1). Across the entire data set and both continents, $78 \%$ of genetic variation existed within populations $\left(F_{\mathrm{ST}}=0.220\right.$; see table 2). The remaining genetic structure was explained primarily by within-continent population differences $\left(F_{\mathrm{SC}}=0.206\right)$, with between-continent differences explaining only $1.7 \%$ of the total variation $\left(F_{\mathrm{CT}}=0.017\right)$.

\section{Tests of Adaptation}

We next tested for a signal of adaptation to the different continents as well as climatic clines while accounting for neutral genetic divergence between sampled populations (table 3). In particular, we implemented a Bayesian mixed model, treating continent as a fixed effect and covariance in phenotype as a random effect that was a function of the genetic similarity between the sampled populations. This approach, similar in principle to the phylogenetic linear mixed models in Grafen (1989), accounted for the covariance in phenotype expected due to similarity at neutral loci while accommodating uncertainty in estimating the signature of adaptation to continent across different traits.
Although the average individual seed mass did not differ between North America and Europe, mean seed mass decreased with increasingly warmer MATs on both continents (fig. $2 A$; table $3 ; P=.030$; table A1; tables A1, A2 available online). Our treatment of seeds was designed to maximize germination, and consequently germination was quite high (80\%) and did not differ between the two continents $\left(F_{1,18}=0.05, P=.828\right)$. Using the combined root and shoot biomass of plants grown in a common environment for 25 days, plants from North America grew faster than plants from Europe, and there was a positive trend between mass and MAT of collection sites on both continents ( $\beta_{\mathrm{MAT}}$; table 3; fig. $2 B$ ). Faster growth of milkweed plants associated with lower latitudes (and higher MAT) is consistent with data from 22 North American populations of $A$. syriaca studied across the entire native range (Woods et al. 2012); the observed continental differentiation of growth beyond these clines is certainly suggestive of adaptation.

Plant growth responded in the opposite direction from seed mass; that is, warmer climates gave rise to plants that grew faster, despite having smaller seeds (fig. $2 A, 2 B$ ). This is consistent with life-history theory, which suggests that smaller seeds should be favored where growth is not limited by environmental factors (Harper 1977). Root growth was strongly differentiated by continent (50\% faster in North America), leading to relatively higher plant allocation to roots versus shoots in North American plants (ta-

Table 3: Phenotypic differentiation among common milkweed populations between continents while accounting for shared evolutionary history (based on putatively neutral genetic divergence)

\begin{tabular}{|c|c|c|c|c|c|c|c|}
\hline Trait & $\beta_{\mathrm{NA}}$ & $\beta_{\mathrm{NA}} 95 \% \mathrm{CI}$ & $\beta_{\mathrm{E}}$ & $\beta_{\mathrm{E}} 95 \% \mathrm{CI}$ & $\beta_{\mathrm{NA}-\mathrm{E}} 95 \% \mathrm{CI}$ & $\beta_{\mathrm{MAT}}$ & $\beta_{\text {MAT }} 95 \%$ CI \\
\hline Seed mass & .12 & $-.682, .914$ & -.065 & $-.872, .694$ & $-.7,1.08$ & -.497 & $-.925,-.063^{a}$ \\
\hline Total mass & .781 & $.0195,1.49$ & -.725 & $-1.46,-.046$ & $.93,2.1^{\mathrm{a}}$ & .138 & $-.16, .443$ \\
\hline Shoot mass & .634 & $-.215,1.43$ & -.599 & $-1.4, .175$ & $.398,1.98^{\mathrm{a}}$ & -.0392 & $-.454, .365$ \\
\hline Root mass & .855 & $.301,1.45$ & -.817 & $-1.39,-.231$ & $1.15,2.17^{a}$ & .103 & $-.148, .353$ \\
\hline Root: shoot & .939 & $.561,1.26$ & -.946 & $-1.32,-.589$ & $1.59,2.17^{\mathrm{a}}$ & .0147 & $-.13, .151$ \\
\hline Root buds & .337 & $-.569,1.18$ & -.333 & $-1.14, .441$ & $-.238,1.58$ & -.0781 & $-.539, .398$ \\
\hline Carbon: nitrogen & .712 & $.0248,1.39$ & -.699 & $-1.4, .041$ & $.756,2.01^{\mathrm{a}}$ & .168 & $-.164, .505$ \\
\hline Trichomes & .278 & $-.526,1.12$ & -.27 & $-1.06, .594$ & $-.454,1.45$ & .17 & $-.314, .666$ \\
\hline Specific leaf area & -.584 & $-1.21, .1$ & .588 & $-.084,1.27$ & $-1.81,-.507^{a}$ & -.429 & $-.794,-.093^{\mathrm{a}}$ \\
\hline Water content & -.418 & $-1.14, .302$ & .452 & $-.245,1.14$ & $-1.65,-.123^{a}$ & -.423 & $-.77,-.042^{\mathrm{a}}$ \\
\hline Monarch mass & -.955 & $-1.29,-.622$ & .936 & $.573,1.29$ & $-2.18,-1.63^{\mathrm{a}}$ & .0276 & $-.112, .163$ \\
\hline Induced resistance & .0739 & $-.778,1$ & -.0525 & $-.957, .82$ & $-.793,1.02$ & .3 & $-.167, .785$ \\
\hline Constitutive latex & -.0734 & $-.878, .816$ & .109 & $-.818,1.04$ & $-1.27, .795$ & -.14 & $-.614, .383$ \\
\hline Induced latex & .299 & $-.529,1.14$ & -.278 & $-1.11, .507$ & $-.309,1.56$ & -.322 & $-.813, .173$ \\
\hline Latex inducibility & .374 & $-.467,1.14$ & -.391 & $-1.17, .39$ & $-.216,1.67$ & -.207 & $-.692, .274$ \\
\hline Constitutive cardenolides & -.92 & $-1.47,-.418$ & .889 & $.382,1.39$ & $-2.24,-1.42^{\mathrm{a}}$ & .0493 & $-.18, .283$ \\
\hline Induced cardenolides & -.751 & $-1.46,-.005$ & .752 & $.015,1.47$ & $-2.17,-.867^{a}$ & .181 & $-.156, .509$ \\
\hline
\end{tabular}

Note: The parameter of interest is the joint marginal distribution of the difference between $\beta_{\mathrm{NA}}$ and $\beta_{\mathrm{E}}$, the phenotypic means estimated for North American $\left(\beta_{\mathrm{NA}}\right)$ and European $\left(\beta_{\mathrm{E}}\right)$ populations. Presented are the $95 \%$ credible intervals (CIs) for each parameter; where the $\beta_{\mathrm{NA}-\mathrm{E}} 95 \%$ CI does not overlap 0, we infer a significant phenotypic difference between the continents (values in bold). In addition, we report $\beta_{\mathrm{MAT}}$, which is the effect size of mean transformed mean annual temperature at the population collection site on the phenotype mean. Significance is similarly determined by the CI. Eleven significant models out of a total of 17 response variables are highly unlikely to occur by chance (binomial expansion test, $P<.001$ ).

${ }^{a}$ Indicates significant phenotypic difference between the continents. 

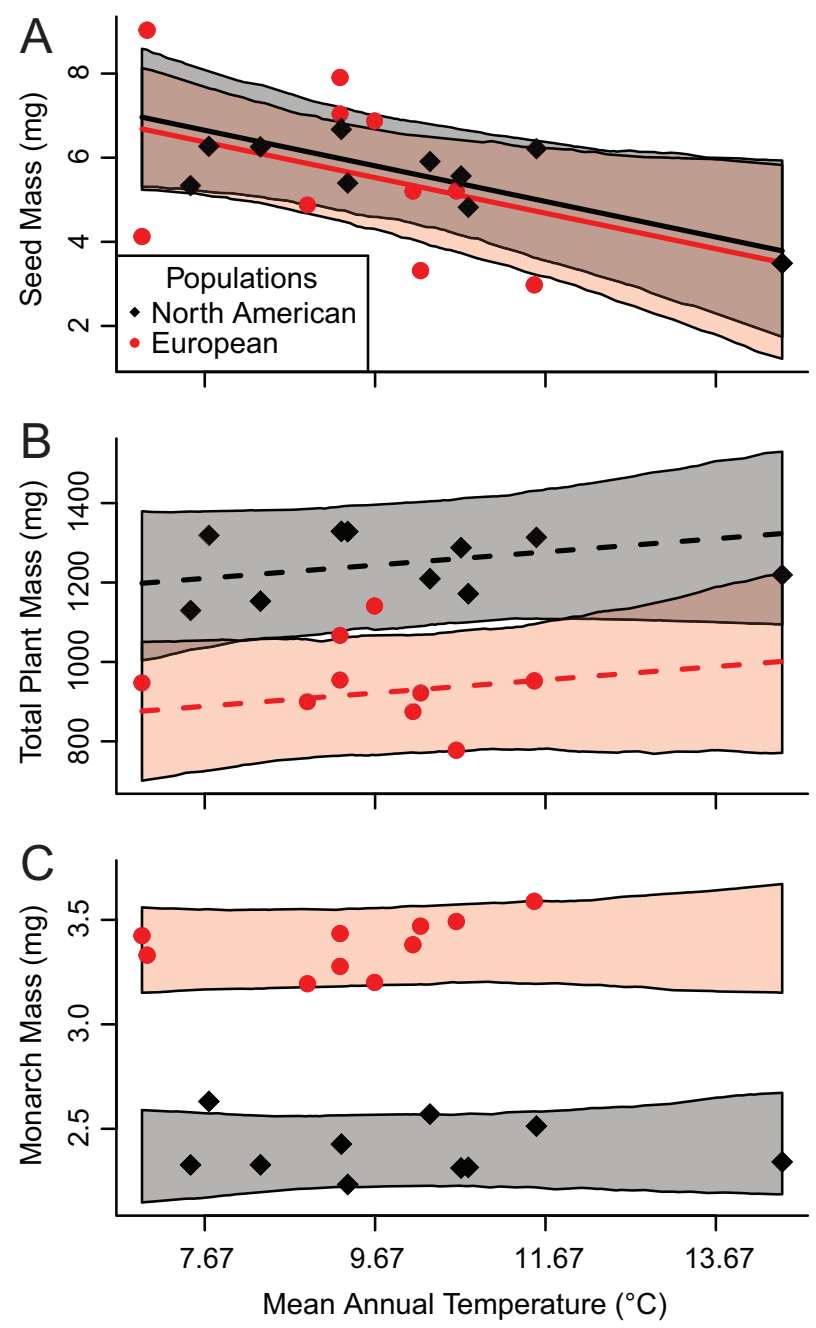

Figure 2: Continental differentiation in individual seed mass $(A)$, total plant mass $(B)$, and plant resistance $(C)$, measured as the growth of monarch caterpillars on common milkweed Asclepias syriaca and also as influenced by mean annual temperature of the collection site. Shown are back-transformed data, with $B$ and $C$ based on common garden measures. Each symbol represents the mean of replicates from a single population from North America or Europe. Shading indicates the $95 \%$ credible interval, and solid line indicates significant relationships between mean annual temperature and the phenotype (dashed line is marginal; in $C$, no line indicates no relationship).

ble 3; fig. 3). Allocation to underground stem-producing root buds did not differ between continents, and MAT of collection sites had only a modest influence on the remaining measured growth parameters (table 3).

Specialist monarch butterfly caterpillars grew $>30 \%$ slower on plants from North America compared with European milkweed populations (table 3; fig. 2C). This loss of resistance is consistent with a release from selection by monarchs in Europe but, surprisingly, was not associated with increased plant growth. The greater susceptibility of
European plants to monarch caterpillars was likely a result of increased food quality, with the 10 European populations having nearly $20 \%$ lower foliar carbon: nitrogen ratio $(\mathrm{C}: \mathrm{N})$ than North American populations (table 3; fig. 3), indicating higher nutrient content. Additionally, the increase in susceptibility of European milkweeds was coupled with $23 \%$ higher constitutive concentrations of cardenolides compared with North American populations (table 3; fig. 4B).

Overall, the analysis of leaf traits suggests that impacts of ecophysiological traits were important. The continental differentiation in $\mathrm{C}: \mathrm{N}$ was driven by a higher nitrogen content of European leaves. In addition, foliar water content and specific leaf area showed a matching pattern, with higher water content $(82.1 \%$ vs. $78.9 \%$; table 3$)$ and reduced leaf density (higher values of SLA) in European milkweed populations (fig. 3 ). Both traits showed declining values with MAT of collection sites (table 3; fig. A2B).

We next assessed a signature of plant adaptation to the two continents using the ratio of $Q_{\mathrm{CT}}$ to $F_{\mathrm{CT}}$, which is analogous to a ratio of $Q_{\mathrm{ST}}$ to $F_{\mathrm{ST}}$ but considers phenotypic and genetic differentiation at the level of the two continents, each with multiple sampled populations (Manier et al. 2007; Whitlock 2008). The results of these analyses mirrored those of the Bayesian analyses (table A2), demon-
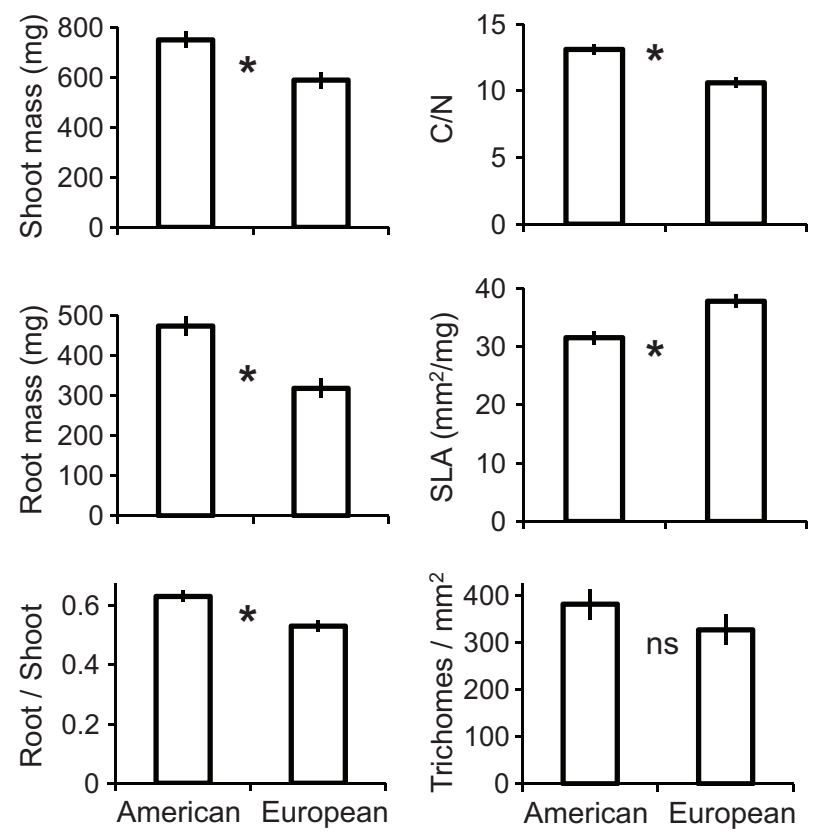

Figure 3: Phenotypic differences (mean \pm SE) between Asclepias syriaca from replicate plants $(n=10$ populations each from North America and Europe) grown in a common environment. Asterisk indicates significance of the continental term at $P<.05$. C/N, ratio of leaf carbon to nitrogen on a dry mass basis; SLA, specific leaf area (area per dry mass). Trichome density was measured on leaf samples. 

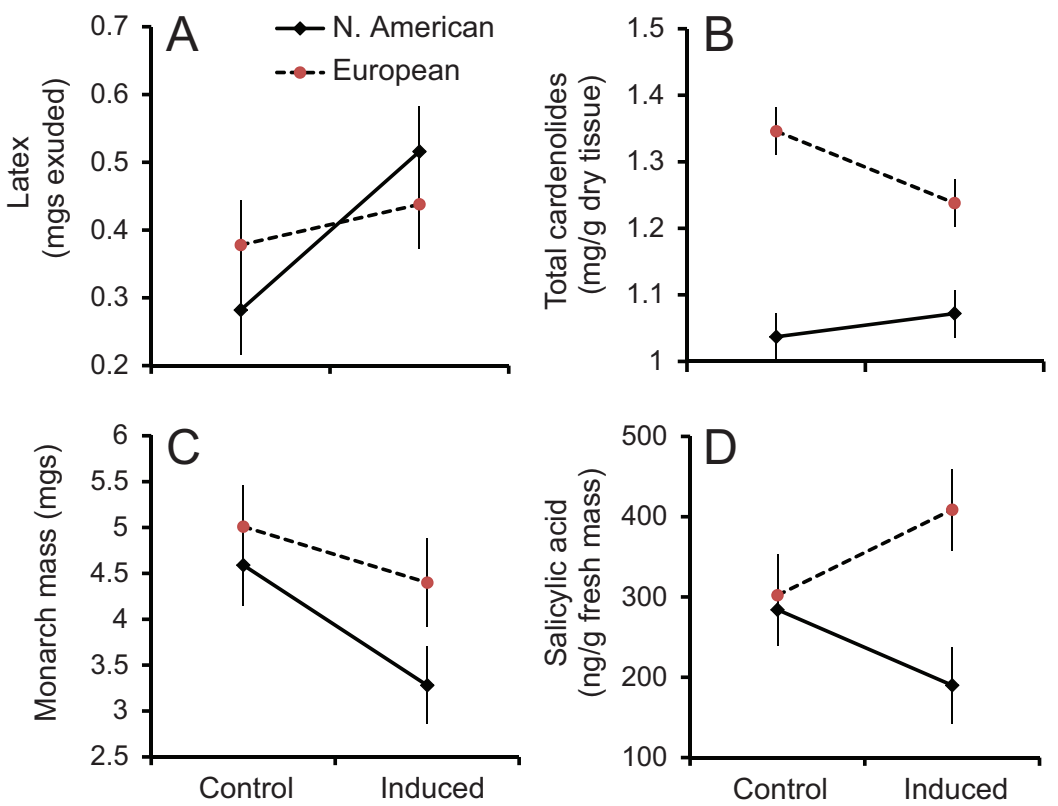

Figure 4: Impacts of continental origin (North America vs. Europe) and herbivore induction by monarch caterpillars on mean \pm SE latex exudation $(A)$, foliar cardenolides $(B)$, mass of subsequently feeding monarch caterpillars $(C)$, and foliar concentrations of salicylic acid $(D)$ in common milkweed Asclepias syriaca grown in a common environment.

strating strong evidence for adaptive differentiation. Additionally, we used $Q_{\mathrm{ST}}: F_{\mathrm{ST}}$ ratios to assess within-continent signatures of adaptation. For North American populations, we found some evidence for stabilizing selection $\left(Q_{\mathrm{ST}}<\right.$ $F_{S T}$ ) on root mass, relative root: shoot allocation, and constitutive leaf cardenolide content, while we found evidence for adaptive differentiation $\left(Q_{\mathrm{ST}}>F_{\mathrm{ST}}\right)$ in leaf physiological traits (SLA, water content, $\mathrm{C}: \mathrm{N}$ ) and resistance to monarch larvae (table A2). Evidence for adaptation among European populations was less evident, with some effects on leaf physiology (e.g., SLA), latex, and resistance to monarch larvae (table A2). Accordingly, the emerging picture is one of adaptive differentiation among native populations but less so among introduced populations.

\section{Inducibility of Defenses and Impacts on Monarch Performance}

The key defensive traits of milkweed-latex and cardenolides-showed continental differences in their inducibility following attack by monarch caterpillars (continent $x$ herbivore interaction for latex: $F_{1,134}=4.103, P=.044$; cardenolides: $F_{1,134}=4.367, P=.039$; fig. $4 A, 4 B$ ). For both types of defense, North American populations showed the typical increase following attack, while European populations showed no increase in the traits (or even a modest decline in cardenolides), indicating genetic differentiation of phenotypic plasticity. Our analysis controlling for neutral molecular differentiation indicated continental differentiation in inducibility of cardenolides but not latex (table 3 ). When we evaluated the effects of these induced responses on the performance of a second, separate set of monarch caterpillars, induction reduced monarch growth by $25 \%$ in North American milkweed populations $\left(F_{1,9.55}=6.610\right.$, $P=.029)$, while in European populations this reduction was only $16 \%$ and not significant $\left(F_{1,4.84}=0.671, P=\right.$ .451; fig. 4C). Yet despite this reduced effect of induction on monarchs in European populations, we did not detect a significant continent $x$ induction interaction for monarch growth (table A1; the inducibility term is not significant in table 3).

\section{Predicting Continental Differences in Inducibility of Cardenolides}

We next evaluated three potential mechanisms underlying the differences in inducibility of cardenolides between the continents, none of which are mutually exclusive: differences in hormonal responses (jasmonic acid and salicylic acid) to damage, plant growth driven differences in inducibility, or trade-offs between constitutive and inducible allocation to defense traits. First, milkweed plants showed a greater than sevenfold increase of jasmonic acid in response to monarch herbivory, and this response was similar between continents (fig. A3). However, salicylic acid, which is typically not induced by chewing herbivores, 
showed a continental difference in induction (continent $x$ herbivore interaction for salicylic acid: $F_{1,27}=4.299, P=$ .048; fig. 4D). Although constitutive salicylic acid concentrations were identical, concentrations were enhanced upon damage by monarchs by $35 \%$ in European plants, whereas North American populations showed a 33\% decline. Second, total plant biomass had a strong effect on cardenolide induction, with larger plants inducing cardenolides more strongly, and this pattern was consistent on both continents (continent: $F_{1,16}=1.341, P=.264$; plant mass: $F_{1,16}=12.156, P=.003$; interaction: $F_{1,16}=1.471, P=$ .243; fig. 5A). Finally, we found a significant trade-off between constitutive and induced allocation to cardenolides in North American populations (corrected correlation and Monte Carlo test, $r=-0.759, P=.048$ ) but not European populations $(r=-0.141, P=.636$; fig. $5 B)$. In the full analysis across all 20 populations, there was a marginal sig-
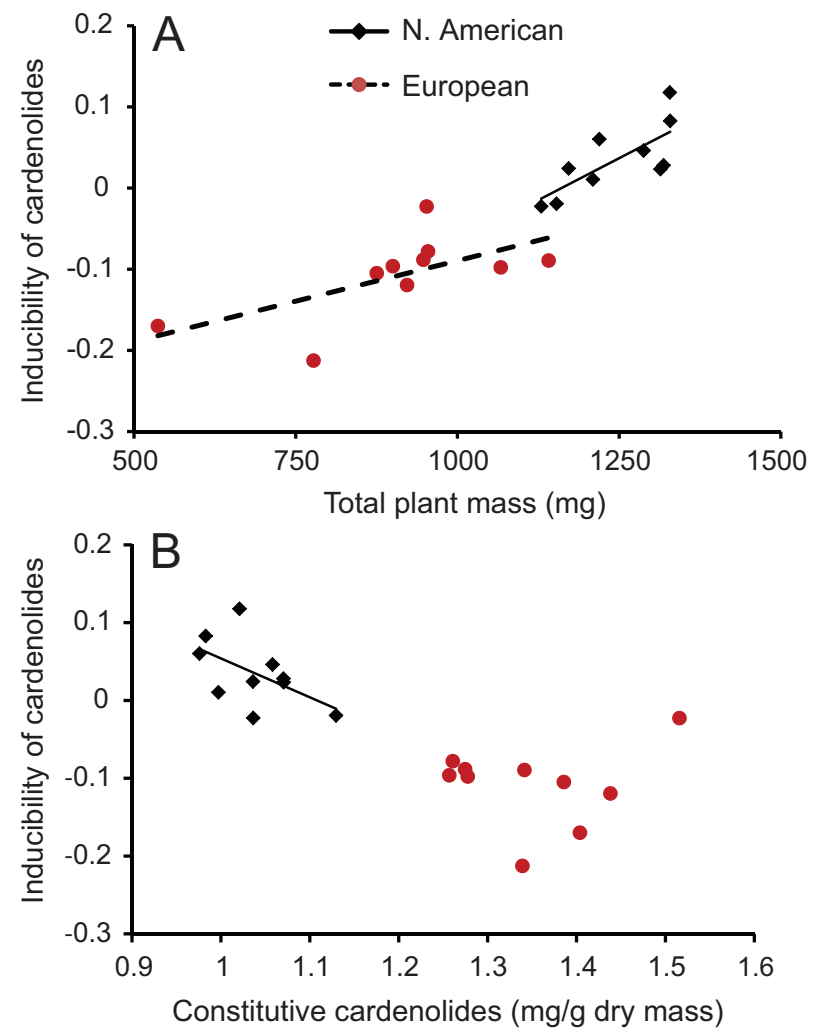

Figure 5: Two predictors of differentially induced cardenolides in North American compared with European populations of common milkweed Asclepias syriaca. A, Plant growth is positively correlated with inducibility in populations from both continents. $B$, A tradeoff between constitutive cardenolides and inducibility is evident only in native North American populations. Note that inducibility of cardenolides is typically positive in North American populations (above 0 on the $Y$-axis) and is largely negative (i.e., reduced concentrations following damage; below 0) in European populations. nal for a trade-off $(r=-0.514, P=.084)$ but no significant difference in the slopes between continents $(P=.157$; two-tailed likelihood that slopes between continents are different, based on the randomization test outlined in Carrillo et al. 2012). It appears that salicylic acid, plant growth, and constitutive cardenolides are independently contributing to the continental differentiation in inducibility, since these traits were not correlated $(P \gg .05$ for the pairwise correlations). Results on the diversity of cardenolides are given in appendix A.

\section{Discussion}

We present several lines of evidence for adaptive divergence in common milkweed populations within its native range and following introduction to Europe some 400 years ago, which is equivalent to a maximum of 125 generations (assuming a minimum generation time of 3 years). The strongest signature of adaptation was for plant growth and leaf physiological traits (SLA and water content), which showed genetically based clines with temperature on both continents and strong continental differentiation after accounting for neutral molecular divergence. Milkweed plants collected from warmer climates grew faster, had a lower specific leaf area (i.e., had denser leaves), and had lower leaf water content. Although we cannot rule out a contribution of maternal effects in generating our measured phenotypes, such effects are unlikely to cause the strong continental differences we observed, given the wide geographic range of populations in both continents and their large overlap in climatic variables. After accounting for variation in the climate of collection sites and neutral molecular variation between populations, these continental differences persisted. Since climatic variables can confound continental comparisons (Colautti et al. 2009), including analyses of clinal variation on each continent is an especially compelling test of adaptation. Because founder effects, genetic drift, and gene flow can impact population differences in genetically based phenotypes, accounting for such history and chance is critical and has been increasingly applied in assessments of adaptation in continental introductions (Chun et al. 2009, 2011; Keller et al. 2009; Xu et al. 2010; Lachmuth et al. 2011). Our results were consistent across both a traditional $Q_{\mathrm{CT}} / F_{\mathrm{CT}}$ test and a multivariate approach that explicitly modeled covariance among populations within and between continents.

We found that both monarch performance and the expression of plant defensive traits (cardenolides and latex) diverged continentally, albeit in complex ways. The decline of resistance to monarchs in European populations is consistent with the lack of this potential selective agent in Europe and appears to be mainly mediated by increased leaf quality (i.e., $\mathrm{C}: \mathrm{N}$ ratio and SLA). Given that latex is 
strongly correlated with monarch performance within and between milkweed species (Zalucki and Malcom 1999; Agrawal 2005; Woods et al. 2012; Agrawal et al. 2015a), the reduced latex induction in European milkweeds may also contribute to reduced resistance to monarchs. In contrast, cardenolides were higher among European milkweed populations, which did not impair growth of monarch caterpillars. Across several past studies, we have not found intraspecific genetic correlations between milkweed traits such as latex, cardenolides, water content, and SLA (e.g., Agrawal 2005; Woods et al. 2012), while in a recent study differences in cardenolides and growth were found to be driving differences in SLA rather than vice versa (Züst et al. 2015). Both lines of evidence suggest that selection on leaf physiology would be an unlikely driver of changes in defensive traits.

Given the established trade-off between constitutive cardenolides and inducibility (Agrawal et al. 2010), we speculate that inducibility of cardenolides might have declined (or even reversed) in response to the selective pressure of Aphis nerii (the only specialist present in Europe) and/or generalist herbivores for high constitutive cardenolide levels. Comparing the hormonal mechanisms of induced responses, European milkweeds showed the typical jasmonic acid burst following monarch herbivory (fig. A2), but they unexpectedly also showed a strong burst of salicylic acid (fig. 4D). Such a hormonal response is more commonly associated with aphid herbivory (Ali and Agrawal 2012) and may therefore represent a shift in the type of plant responses to the dominant herbivore in Europe (Bukovinszky et al. 2014).

While we have focused on two major herbivores of milkweed, several other specialist insects exclusive to the native range of milkweed are highly damaging, feed on different plant parts (Agrawal 2005), and are thus likely to respond to resistance traits differently. Accordingly, future work may focus on community interactions between multiple herbivores and plants from each continent, since this might identify key drivers and traits in herbivore-host plant specialization. In sum, although the evolutionary driver of higher cardenolides and reduced inducibility in introduced milkweed populations is unclear, both physiological and ecological mechanisms are consistent with adaptive explanations, and the magnitude of these patterns was not explained by neutral genetic divergence or patterns of temperature-related climate variables or precipitation.

A burst of recent studies has compared inducibility among native and introduced plant populations (Cipollini et al. 2005; Eigenbrode et al. 2008; Beaton et al. 2011; Carrillo et al. 2012; Wang et al. 2012, 2013; Gu et al. 2014). Beaton et al. (2011) compared an invasive genotype of Chinese bushclover (Lespedeza cuneata) with both an ancestral genotype from the time of first introduction and a genotype from the native range of the species, and they found that the invasive genotype had the lowest constitutive defense but was the most inducible. Similarly, Cipollini et al. (2005) demonstrated that three out of four nonnative North American populations of garlic mustard (Alliaria petiolata) showed lower constitutive levels and higher inducibility of glucosinolates than European collections. Carrillo et al. (2012) recently reported that a set of indirect defensive traits associated with extrafloral nectaries of the tallow tree were reduced in populations from the introduced compared with native range; despite a lack of continental differences in inducibility of extrafloral nectar, they did find a trade-off between constitutive and induced nectary production (and this trade-off was consistent in populations from the two continents). The only other study to assess latex in native and exotic plants (Gu et al. 2014) reported stronger latex induction in invasive populations of the tallow tree in response to one herbivore but not another. Our results are only somewhat consistent with these findings, indicative of relaxed selection for defense (i.e., improved monarch performance) in the invaded range but more complex altered dynamics of latex, cardenolides, and their inducibility. Nonetheless, these studies and ours were consistent with plant defense theory that predicts a trade-off between constitutive and induced defense within a species (Brody and Karban 1992; Agrawal et al. 2010).

Overall, Eigenbrode et al. (2008) have argued that although constitutive and induced resistance may not vary consistently across continents, the founder effects associated with plant introductions should give rise to higher variance in the inducibility of introduced populations, even in the absence of adaptive evolution. Accordingly, the most robust way to understand continental differences in any phenotype is to combine common garden studies, assessments of population genetic structure, and signatures of selection. In our case, although founder effects may have given rise to high $F_{\mathrm{ST}}$ values among populations in Europe, we did not find evidence for high phenotypic variability among populations. On the contrary, there appears to be convergence in phenotypes among European populations.

\section{Conclusion}

Our results are consistent with population-level adaptation to geographic variation in the biotic and/or abiotic environment within continents and, to a greater extent, across continents where community interactions are highly altered (Thompson 2005; Berenbaum and Zangerl 2006; Zangerl and Berenbaum 2007). Although an evolutionary relaxation of defense investment is often predicted when plants are introduced to a new environment, nature is rarely so simple (Blossey and Nötzold 1995; Bossdorf et al. 2005; Stastny et al. 2005). Differences in climate, specialist versus 
generalist herbivores, and plant population structure conspire to shape selection and potentially constrain adaptation on multiple traits. Additionally, because plant defenses are frequently inducible, responses to selection may be missed when studied in the absence of herbivores. The results for common milkweed indicate a clear pattern of reduced inducibility of cardenolides in European populations compared with their North American ancestors. This reduced inducibility may have been driven by the specific herbivores present and absent in Europe, trade-offs between constitutive and inducible defenses, and/or selection on growth strategies and primary metabolism. We speculate that these evolutionary changes in plant defense expression occurred independently of the evolution of adaptations to the novel abiotic environment in Europe.

\section{Acknowledgments}

For collecting seeds in North America, we are grateful to C. Delphia, P. Dubin, S. Gallaher, B. Irwin, M. Johnson, H. Michaels, P. Van Zandt, E. Wurtz, and the late A. Zangerl; in Europe, we thank I. Bereczki, M. Marotti, P. Mižík, C. Roge, G. Szerényi, and M. Valachovič. We thank S. Arnold, R. Bell, J. Berg, M. Hare, and M. Manier for assistance and advice on population genetic analyses. Molecular work was conducted in the Evolutionary Genetics Core Facility at Cornell University, with support from S. Bogdanowicz. All chemical analyses were conducted in the Cornell Chemical Ecology Group's Core Facility, with help from R. Halitschke. S. Rasmann helped with statistical analyses of trade-offs between constitutive and induced cardenolides. We thank anonymous reviewers, B. Blossey, M. Geber, M. Hare, S. Kalisz, L. Martin, M. Weber, A. Winn, and the phytophagy lab (http://www.herbivory.com) for discussion or comments on the manuscript. This research was supported by NSF-DEB 1118783 to A.A.A., Research Experiences for Undergraduates supplements that supported E.C.W., Swiss National Science Foundation grant PBZHP3-141434 to T.Z., and the John Templeton Foundation.

\section{Literature Cited}

Agrawal, A. A. 2005. Natural selection on common milkweed (Asclepias syriaca) by a community of specialized insect herbivores. Evolutionary Ecology Research 7:651-667.

Agrawal, A. A., J. G. Ali, S. Rasmann, and M. Fishbein. 2015a. Macroevolutionary trends in the defense of milkweeds against monarchs: latex, cardenolides, and tolerance of herbivory. Pages 47-59 in $\mathrm{K}$. Oberhauser, ed. The monarch butterfly: biology and conservation. Cornell University Press, Ithaca, NY.

Agrawal, A. A., J. K. Conner, and S. Rasmann. 2010. Tradeoffs and adaptive negative correlations in evolutionary ecology. Pages
243-268 in M. Bell, W. Eanes, D. Futuyma, and J. Levinton, eds. Evolution after Darwin: the first 150 years. Sinauer, Sunderland, MA.

Agrawal, A. A., A. Hastings, G. Bradburd, E. Woods, T. Zust, J. Harvey, and T. Bukovinszky. 2015b. Data from: Evolution of plant growth and defense in a continental introduction. American Naturalist, Dryad Digital Repository, http://dx.doi.org/10.5061/dryad .vr176.

Agrawal, A. A., and K. Konno. 2009. Latex: a model for understanding mechanisms, ecology, and evolution of plant defense against herbivory. Annual Review of Ecology, Evolution, and Systematics 40:311-331.

Agrawal, A. A., G. Petschenka, R. A. Bingham, M. G. Weber, and S. Rasmann. 2012. Toxic cardenolides: chemical ecology and coevolution of specialized plant-herbivore interactions. New Phytologist 194:28-45.

Ali, J. G., and A. A. Agrawal. 2012. Specialist versus generalist insect herbivores and plant defense. Trends in Plant Science 17: 293-302.

Barrett, S. C. H., and B. C. Husband. 1990. Genetics of plant migration and colonization. Pages 254-277 in A. H. D. Brown, M. T. Clegg, A. L. Kahler, and B. S. Weir, eds. Plant population genetics, breeding and genetic resources. Sinauer, Sunderland, MA.

Beaton, L. L., P. A. Van Zandt, E. J. Esselman, and T. M. Knight. 2011. Comparison of the herbivore defense and competitive ability of ancestral and modern genotypes of an invasive plant, Lespedeza cuneata. Oikos 120:1413-1419.

Berenbaum, M. R., and A. R. Zangerl. 2006. Parsnip webworms and host plants at home and abroad: trophic complexity in a geographic mosaic. Ecology 87:3070-3081.

Bingham, R. A., and A. A. Agrawal. 2010. Ecological genetics of herbivore-specific induced defenses in common milkweed. $\underline{\text { Jour- }}$ nal of Ecology 98:1014-1022.

Blossey, B., and R. Nötzold. 1995. Evolution of increased competitive ability in invasive nonindigenous plants: a hypothesis. $\underline{\text { Journal of }}$ Ecology 83:887-889.

Bossdorf, O., H. Auge, L. Lafuma, W. E. Rogers, E. Siemann, and D. Prati. 2005. Phenotypic and genetic differentiation between native and introduced plant populations. Oecologia (Berlin) 144: $1-11$.

Brody, A. K., and R. Karban. 1992. Lack of a tradeoff between constitutive and induced defenses among varieties of cotton. Oikos 65:301-306.

Bukovinszky, T., R. Gols, A. A. Agrawal, C. Roge, T. M. Bezemer, A. Biere, and J. A. Harvey. 2014. Reciprocal interactions between native and introduced populations of common milkweed, Asclepias syriaca, and the specialist aphid, Aphis nerii. Basic and Applied Ecology 15:444-452.

Callaway, R. M., G. C. Thelen, A. Rodriguez, and W. E. Holben. 2004. Soil biota and exotic plant invasion. Nature 427:731-733.

Carrillo, J., Y. Wang, J. Q. Ding, K. Klootwyk, and E. Siemann. 2012. Decreased indirect defense in the invasive tree, Triadica sebifera. Plant Ecology 213:945-954.

Chun, Y. J., V. Le Corre, and F. Bretagnolle. 2011. Adaptive divergence for a fitness-related trait among invasive Ambrosia artemisifolia populations in France. Molecular Ecology 20:1378-1388.

Chun, Y. J., J. D. Nason, and K. A. Moloney. 2009. Comparison of quantitative and molecular genetic variation of native vs. invasive populations of purple loosestrife (Lythrum salicaria L., Lythraceae). Molecular Ecology 18:3020-3035. 
Cipollini, D., J. Mbagwu, K. Barto, C. Hillstrom, and S. Enright. 2005. Expression of constitutive and inducible chemical defenses in native and invasive populations of Alliaria petiolata. Journal of Chemical Ecology 31:1255-1267.

Colautti, R. I., J. L. Maron, and S. C. H. Barrett. 2009. Common garden comparisons of native and introduced plant populations: latitudinal clines can obscure evolutionary inferences. Evolutionary Applications 2:187-199.

Dieringer, D., and C. Schlotterer. 2003. Microsatellite analyser (MSA): a platform independent analysis tool for large microsatellite data sets. Molecular Ecology Notes 3:167-169.

Eigenbrode, S. D., J. E. Andreas, M. G. Cripps, H. Ding, R. C. Biggam, and M. Schwarzlander. 2008. Induced chemical defenses in invasive plants: a case study with Cynoglossum officinale L. Biological Invasions 10:1373-1379.

Evanno, G., S. Regnaut, and J. Goudet. 2005. Detecting the number of clusters of individuals using the software STRUCTURE: a simulation study. Molecular Ecology 14:2611-2620.

Excoffier, L., and H. E. L. Lischer. 2010. Arlequin suite ver 3.5: a new series of programs to perform population genetics analyses under Linux and Windows. Molecular Ecology Resources 10:564567.

Gaertner, E. E. 1979. History and use of milkweed (Asclepias syriaca L). Economic Botany 33:119-123.

Grafen, A. 1989. The phylogenetic regression. Philosophical Transactions of the Roval Society B: Biological Sciences 326:119-157.

Gu, X., E. Siemann, L. Zhu, S. X. Gao, Y. Wang, and J. Q. Ding. 2014. Invasive plant population and herbivore identity affect latex in duction. Ecological Entomology 39:1-9.

Harper, J. L. 1977. Population biology of plants. Academic Press, London.

Jakobsson, M., and N. A. Rosenberg. 2007. CLUMPP: a cluster matching and permutation program for dealing with label switching and multimodality in analysis of population structure. Bioinformatics 23:1801-1806.

Kabat, S. M., C. W. Dick, and M. D. Hunter. 2010. Isolation and characterization of microsatellite loci in the common milkweed, Asclepias syriaca (Apocynaceae). American Journal of Botany 97: E37-E38.

Keller, S. R., D. R. Sowell, M. Neiman, L. M. Wolfe, and D. R. Taylor. 2009. Adaptation and colonization history affect the evolution of clines in two introduced species. New Phytologist 183:678-690.

Kolbe, J. J., M. Leal, T. W. Schoener, D. A. Spiller, and J. B. Losos. 2012. Founder effects persist despite adaptive differentiation: a field experiment with lizards. Science 335:1086-1089.

Lachmuth, S., W. Durka, and F. M. Schurr. 2011. Differentiation of reproductive and competitive ability in the invaded range of Senecio inaequidens: the role of genetic Allee effects, adaptive and nonadaptive evolution. New Phvtologist 192:529-541.

Manier, M. K., C. M. Seyler, and S. J. Arnold. 2007. Adaptive divergence within and between ecotypes of the terrestrial garter snake, Thamnophis elegans, assessed with F(ST)-Q(ST) comparisons. Iournal of Evolutionary Biology 20:1705-1719.

Maron, J. L., M. Vila, R. Bommarco, S. Elmendorf, and P. Beardsley. 2004. Rapid evolution of an invasive plant. Ecological Monographs 74:261-280.

Montague, J. L., S. C. H. Barrett, and C. G. Eckert. 2008. Reestablishment of clinal variation in flowering time among introduced populations of purple loosestrife (Lythrum salicaria, Lythraceae). Journal of Evolutionary Biology 21:234-245.
Morris, W. F., M. B. Traw, and J. Bergelson. 2006. On testing for a tradeoff between constitutive and induced resistance. Oikos 112:102-110.

O'Quinn, R. L., and M. Fishbein. 2009. Isolation, characterization and cross-species amplification of polymorphic microsatellite loci in Asclepias (Apocynaceae). Conservation Genetics 10:1437-1440.

Orians, C. M., and D. Ward. 2010. Evolution of plant defenses in nonindigenous environments. Annual Review of Entomology 55: 439-459.

Ovaskainen, O., M. Karhunen, C. Zheng, J. M. C. Arias, and J. Merilä. 2011. A new method to uncover signatures of divergent and stabilizing selection in quantitative traits. Genetics 189:621-632.

Pritchard, J. K., M. Stephens, and P. Donnelly. 2000. Inference of population structure using multilocus genotype data. Genetics 155:945-959.

Rasmann, S., and A. A. Agrawal. 2011. Latitudinal patterns in plant defense: evolution of cardenolides, their toxicity and induction following herbivory. Ecology Letters 14:476-483.

R Development Core Team. 2007. R: a language and environment for statistical computing. R Foundation for Statistical Computing, Vienna. http://www.R-project.org.

Rosenberg, N. A. 2004. DISTRUCT: a program for the graphical display of population structure. Molecular Ecology Notes 4:137-138.

Sakai, A. K., F. W. Allendorf, J. S. Holt, D. M. Lodge, J. Molofsky, K. A. With, S. Baughman, et al. 2001. The population biology of invasive species. Annual Review of Ecology and Systematics 32:305-332.

Sax, D. F., J. J. Stachowicz, and S. D. Gaines. 2005. Species invasions: insights into ecology, evolution and biogeography. Sinauer, Sunderland, MA.

Stastny, M., U. Schaffner, and E. Elle. 2005. Do vigour of introduced populations and escape from specialist herbivores contribute to invasiveness? Journal of Ecology 93:27-37.

Thaler, J. S., A. A. Agrawal, and R. Halitschke. 2010. Salicylatemediated interactions between pathogens and herbivores. Ecology 91:1075-1082.

Thompson, J. 2005. The geographic mosaic of coevolution. University of Chicago Press, Chicago.

Visscher, P. M., S. E. Medland, M. A. Ferreira, K. I. Morley, G. Zhu, B. K. Cornes, G. W. Montgomery, and N. G. Martin. 2006. Assumptionfree estimation of heritability from genome-wide identity-by-descent sharing between full siblings. PLoS Genetics 2:e41.

Wang, Y., J. Carrillo, E. Siemann, G. S. Wheeler, L. Zhu, X. Gu, and J. Q. Ding. 2013. Specificity of extrafloral nectar induction by herbivores differs among native and invasive populations of tallow tree. Annals of Botany 112:751-756.

Wang, Y., E. Siemann, G. S. Wheeler, L. Zhu, X. Gu, and J. Q. Ding. 2012. Genetic variation in anti-herbivore chemical defences in an invasive plant. Journal of Ecology 100:894-904.

Whitlock, M. C. 2008. Evolutionary inference from Q(ST). Molecular Ecology 17:1885-1896.

Wilson, A. J., D. Reale, M. N. Clements, M. M. Morrissey, E. Postma, C. A. Walling, L. E. Kruuk, and D. H. Nussey. 2010. An ecologist's guide to the animal model. Lournal of Animal Ecology 79: 13-26.

Woods, E. C., A. P. Hastings, N. E. Turley, S. B. Heard, and A. A. Agrawal. 2012. Adaptive geographical clines in the growth and defense of a native plant. Ecological Monographs 82:149-168.

Xu, C. Y., M. H. Julien, M. Fatemi, C. Girod, R. D. Van Klinken, C. L. Gross, and S. J. Novak. 2010. Phenotypic divergence during the invasion of Phyla canescens in Australia and France: evidence for selection-driven evolution. Ecology Letters 13:32-44. 
Zalucki, M. P., and S. B. Malcolm. 1999. Plant latex and firstinstar monarch larval growth and survival on three North American milkweed species. Journal of Chemical Ecology 25:18271842.

Zangerl, A. R., and M. R. Berenbaum. 2007. Increase in toxicity of an invasive weed after reassociation with its coevolved herbivore. Proceedings of the National Academv of Sciences of the USA 104:12228-12228.
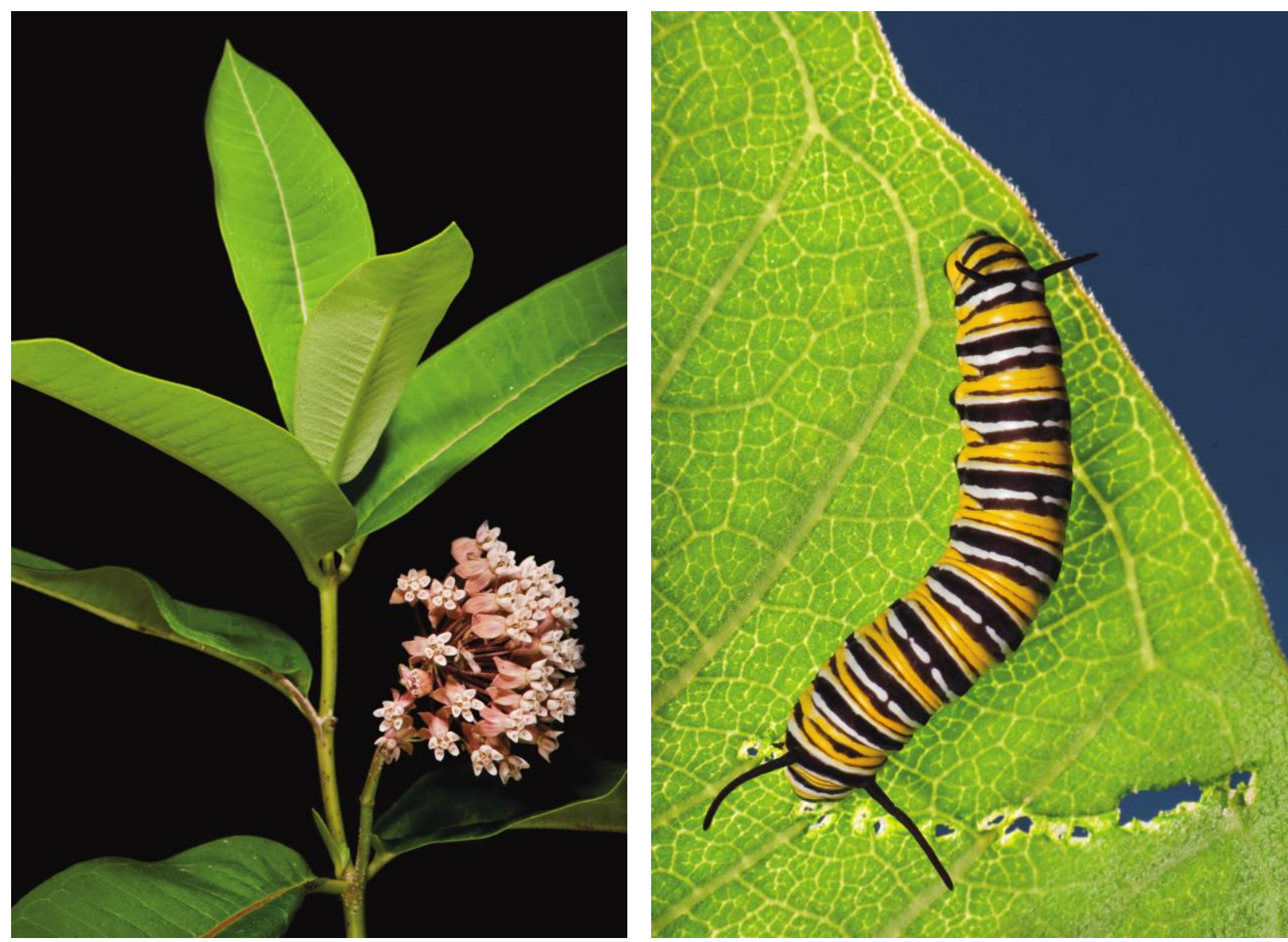

Left, the common milkweed (Asclepias syriaca). Right, a monarch butterfly caterpillar (Danaus plexippus). Photo credits: Ellen C. Woods. 